\title{
Validation of the Graded Prognostic Assessment for Melanoma Using Molecular Markers (Melanoma-molGPA)
}

\author{
Carsten Nieder ${ }^{\mathrm{a}, \mathrm{b}}$, e, Mandy Hintz ${ }^{\mathrm{c}}$, Angelika Bilger ${ }^{\mathrm{c}}$, Oliver Oehlke ${ }^{\mathrm{c}}$, Anca-Ligia Grosu ${ }^{\mathrm{c}, \mathrm{d}}$
}

\begin{abstract}
Background: It has been suggested to replace the diagnosis-specific graded prognostic assessment (DS-GPA, based on performance status and number of brain metastases) for patients with primary malignant melanoma with the new Melanoma-molGPA. The latter is a more complex assessment, which also includes BRAF mutation status, age and extracranial metastases. To test the performance of the Melanoma-molGPA, we performed a validation study of this new survival prediction tool.
\end{abstract}

Methods: A retrospective analysis of patients treated at two different academic institutions was performed. The four-tiered MelanomamolGPA was calculated as suggested in the original study.

Results: Median overall survival was 5.4 months ( $95 \%$ confidence interval: 3.1 - 7.7 months). Median survival in the four prognostic classes was $2.1,7.8,11.8$, and 18.0 months, respectively. The 1 -year survival rates were $3 \%, 25 \%, 43 \%$, and $80 \%$, respectively. The difference between the Kaplan-Meier curves was significant $(\mathrm{P}=0.0001, \log$-rank test).

Conclusions: The present survival outcomes support the use of the Melanoma-molGPA. However, survival was better in each of the four groups in the original study. Possible reasons include lead-time bias and different treatment policies.

Keywords: Brain metastases; Melanoma; Radiotherapy; Radiosurgery; Prognostic score

\section{Introduction}

Brain metastases from malignant melanoma are often associ-

Manuscript submitted November 1, 2017, accepted November 21, 2017

aDepartment of Oncology and Palliative Medicine, Nordland Hospital, 8092 Bodo, Norway

${ }^{b}$ Institute of Clinical Medicine, Faculty of Health Sciences, University of Tromso, 9037 Tromso, Norway

'Department of Radiation Oncology, University Medical Center Freiburg, Medical Faculty Freiburg, Robert-Koch-Str.3, Freiburg, Germany

${ }^{\mathrm{d}}$ German Cancer Consortium (DKTK), Partner Site Freiburg, Germany

${ }^{\mathrm{e} C}$ Corresponding Author: Carsten Nieder, Department of Oncology and Palliative Medicine, Nordland Hospital, 8092 Bodo, Norway.

Email: cnied@hotmail.com ated with a short remaining lifespan $[1,2]$. However, a small proportion of patients have repeatedly been reported to survive for several years, rather than just a few months [3-7]. In the present era of multiple local and systemic treatment options, the challenge is to assign the right patient to the right treatment [8], with clear objectives set upfront, e.g., temporary palliation of symptoms or local tumor ablation, which potentially might result in long-term survival.

Independently validated prognostic models might guide clinical decision making [9]. Sperduto et al have published a series of analyses that resulted in widely used scores. Initially, they evaluated data from five randomized Radiation Therapy Oncology Group (RTOG) trials on treatment of brain metastases [10]. They proposed a score that was named graded prognostic assessment (GPA). In the GPA system, three different values $(0,0.5$ or 1$)$ were assigned for each of these four parameters: age ( $\geq 60 ; 50-59 ;<50$ years), Karnofsky performance status (KPS, $<70 ; 70-80 ; 90-100$ ), number of brain metastases $(>3 ; 2-3 ; 1)$, and extracranial metastases (present; not applicable; none). The patients with the best prognosis would have a GPA of 4.0 .

Later, they created diagnosis-specific GPA scores (DSGPA), e.g. for patients with primary malignant melanoma [11]. In the latter group, which included 483 patients, only two factors were significantly associated with survival, KPS and number of brain metastases. The authors assigned two points for KPS 90 100 and single brain metastasis. One point was assigned for KPS $70-80$ and $2-3$ brain metastases. Also in this scoring system, the patients with the best prognosis would have a GPA of 4.0.

Recently, the melanoma DS-GPA was modified, because it had been shown that a molecular marker (BRAF mutation) also influenced survival [12]. The multi-institutional retrospective database included 823 melanoma patients with newly diagnosed brain metastases from January 1, 2006 to December 31,2015 . There were five significant prognostic factors for survival (age, KPS, extracranial metastases, number of brain metastases, and BRAF status). Median survival was 9.8 months. The median survival times for patients with Melanoma-molGPA of $0-1.0,1.5-2.0,2.5-3.0$, and $3.5-4.0$ were $4.9,8.3,15.8$, and 34.1 months $(\mathrm{P}<0.0001)$. It is important to validate this model in other databases. Therefore, the present analysis was performed.

\section{Materials and Methods}

We analyzed patients from a previously described brain me- 
Table 1. Calculation of the Prognostic Score, as Reported by Sperduto et al [12]

\begin{tabular}{llll}
\hline Parameter & 0 points & $\mathbf{0 . 5}$ points $\mathbf{1}$ point \\
\hline $\begin{array}{l}\text { Age } \geq 70 \text { years } \\
\text { Age }<70 \text { years }\end{array}$ & $\mathrm{x}$ & $\mathrm{x}$ & \\
KPS $\leq 70$ & $\mathrm{x}$ & & \\
KPS 80 & & $\mathrm{x}$ & \\
KPS $90-100$ & & $\mathrm{x}$ \\
Presence of extracranial metastases & $\mathrm{x}$ & & \\
$\begin{array}{l}\text { Absence of extracranial metastases } \\
\text { More than four brain metastases }\end{array}$ & $\mathrm{x}$ & & $\mathrm{x}$ \\
$2-4$ brain metastases & & $\mathrm{x}$ & \\
$\begin{array}{l}1 \text { brain metastasis } \\
\text { Negative/unknown BRAF status }\end{array}$ & $\mathrm{x}$ & & $\mathrm{x}$ \\
Positive BRAF status & & $\mathrm{x}$ & \\
\hline
\end{tabular}

tastases database, which is maintained and updated at the first author's institution $[13,14]$. The patients were treated at two different academic institutions in Germany and Norway. For this retrospective study, the patients with primary malignant melanoma treated between 2006 and 2016 were selected $(\mathrm{n}=$ 69 after exclusion of patients managed with best supportive care alone). Treatment was highly individualized and included surgery, local and/or whole-brain radiotherapy (WBRT), and salvage with repeat surgery and/or radiotherapy. Sequential systemic therapy was at the discretion of the medical oncologists. Actuarial survival was calculated with the Kaplan-Meier method and compared between different groups with the log- rank test. Four patients were alive at last recorded follow-up and censored in the actuarial survival analyses. Date of death was entered in all other patients. The median follow-up was 5.5 months. The prognostic impact of all parameters included in the Melanoma-molGPA was tested in univariate analyses (log-rank test). A P-value $\leq 0.05$ was considered statistically significant. The Melanoma-molGPA was calculated as suggested by Sperduto et al [12] and shown in Table 1, i.e. based on age, KPS, presence of extracranial metastases, number of brain metastases and BRAF status. In addition, the previous GPA was calculated as suggested by Sperduto et al [11] (2 points for KPS 90 - 100 and single brain metastasis; 1 point for KPS 70 - 80 and 2 - 3 brain metastases).

All procedures followed were in accordance with the ethical standards of the responsible committee on human experimentation (institutional and national) and with the Helsinki Declaration of 1975 (in its most recently amended version).

\section{Results}

Eight patients $(12 \%)$ had brain metastases already when they were diagnosed with malignant melanoma, and the others had metachronous metastases. In 16 cases $(23 \%)$, the time interval was longer than 5 years. Forty-eight patients $(70 \%)$ were managed with WBRT alone, the others with surgery, radiosurgery (RS) fractionated stereotactic radiotherapy and combinations thereof, sometimes supplemented with WBRT. The fractionation schedules were at the discretion of the treating radiation oncologists. The median age was 61 years, ranging $24-90$. The BRAF status was recorded in only 21 patients $(30 \%)$. Table 2 shows the baseline characteristics and their impact on overall survival, stratified in the same way as in the study by

Table 2. Pretreatment Characteristics of All 69 Patients Included in This Study

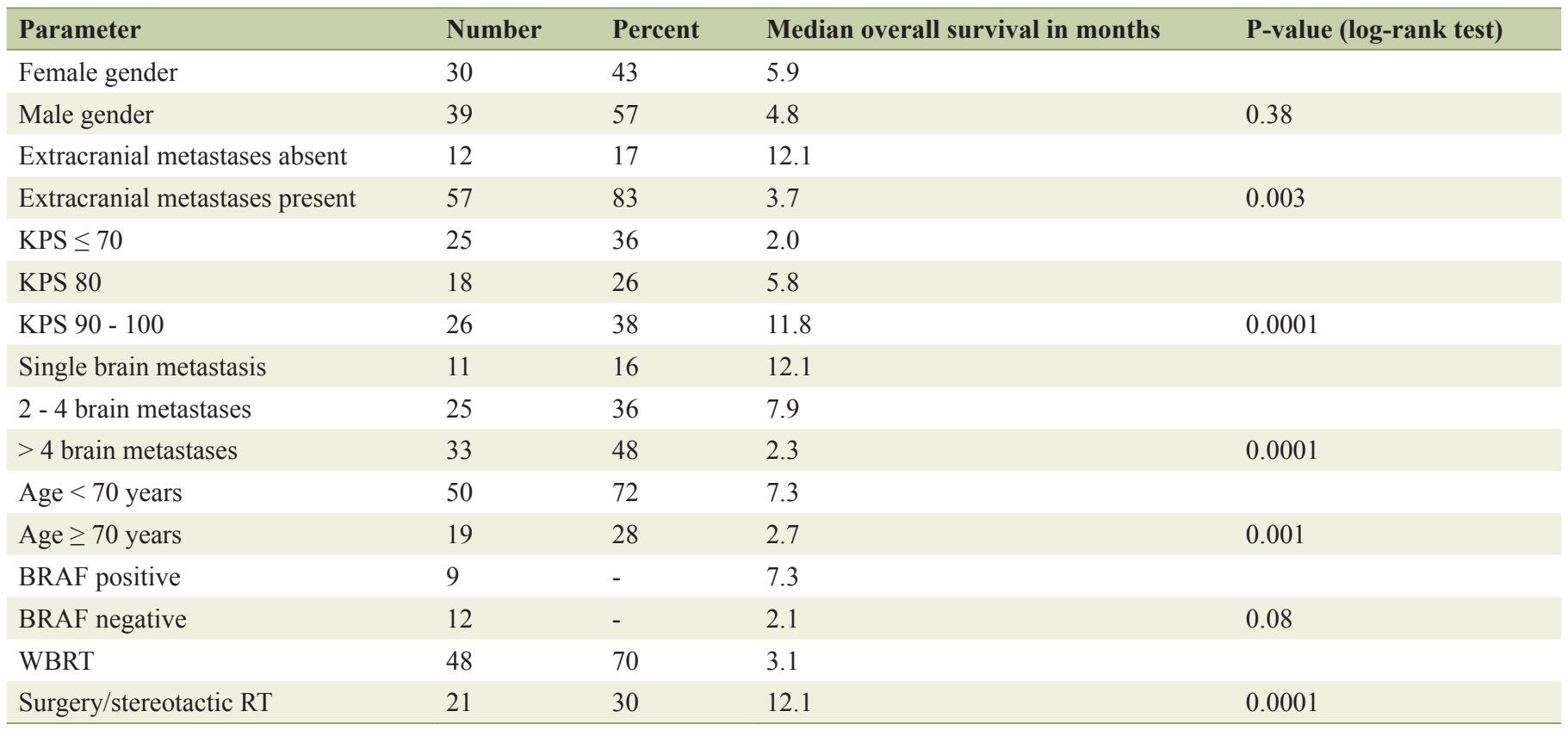

KPS: Karnofsky performance status; WBRT: whole-brain radiotherapy. 


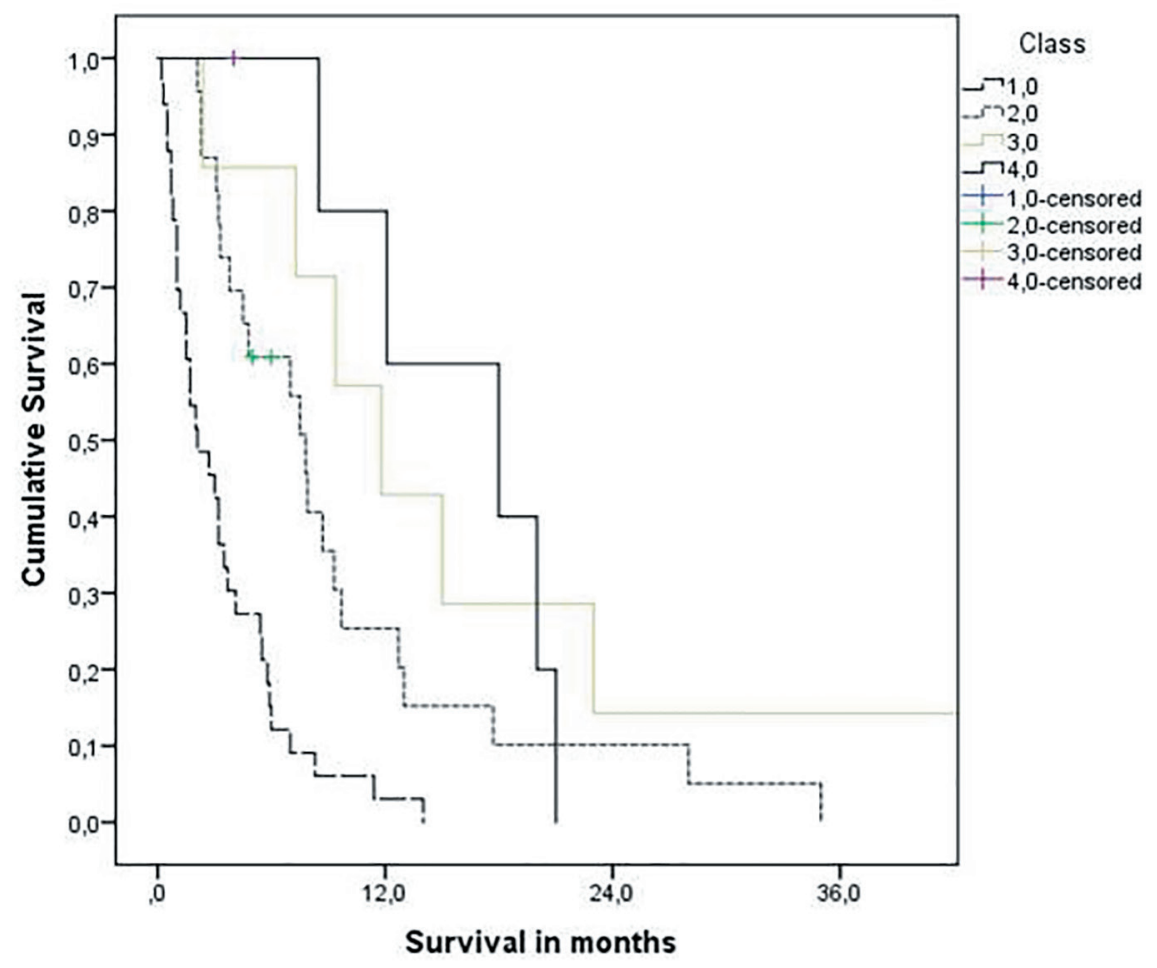

Figure 1. Kaplan-Meier curves for overall survival: molGPA $4.0(3.5-4$ points, $n=6)$ vs. molGPA $3.0(2.5-3$ points, $n=7)$ vs. molGPA 2.0 (1.5 - 2 points, $n=23)$ and molGPA $1.0(0-1$ points, $n=33), P=0.0001$ (pooled over all strata).

Sperduto et al [12]. Median overall survival was 5.4 months (95\% confidence interval: 3.1 - 7.7 months).

The Melanoma-molGPA score was 0 - 4 . Very few patients were assigned to the two favorable prognostic groups (six patients had 3.5 - 4 points and seven had 2.5 - 3 points). Figure 1 displays the actuarial survival curves for all four prognostic strata. Median survival was 2.1, 7.8, 11.8, and 18.0 months. The 1 -year rates were $3 \%, 25 \%, 43 \%$, and $80 \%$. In contrast, separation between the four groups was worse with the old model (median survival 2.1, 4.5, 23.0 and 15.0 months; 1 -year rates $6 \%, 5 \%, 64 \%$ and $86 \%$ ). There was a lack of significant separation between the two poor prognostic groups as well as between the two good prognostic groups.

\section{Discussion}

Historically, the melanoma DS-GPA has proven useful, despite the fact that age and extracranial metastases were not part of this score [11]. The prediction model was developed in a multi-institutional analysis of 483 patients treated with different approaches between 1985 and 2007. Median survival was 2.9 months after WBRT (3.1 months in our previous study [3], 3.1 months in the present study). Significantly longer survival was consistently found after RS and/or surgery with or without WBRT [3, $5,6,11,12$, present results]. As different treatment options are available, development of tools that might guide clinicians is of great importance. Sperduto et al have suggested to implement the new four-tiered Melanoma-molGPA [12], a much more comprehensive model than their previous one. Most notably, it also includes a molecular marker (BRAF) with profound impact on systemic treatment paradigms [8]. In line with Sperduto et al, other groups have shown that survival is longer if BRAF mutations are detectable $[15,16]$. The same result was obtained in the present study, albeit in a very small group of patients and therefore without statistical significance $(\mathrm{P}=0.08)$.

In general, the number of patients in our study was limited and so was the statistical power. It is nevertheless, to the best of our knowledge, the first attempt to validate the MelanomamolGPA in an independent dataset. In addition, the study was performed in a different geographical region (Europe), meaning that slightly different diagnostic and therapeutic pathways were employed. For example, brain imaging in asymptomatic patients (screening) was uncommon in our population. If BRAF inhibitors are initiated after brain metastases diagnosis in a screening-detected population, survival will be much longer than in patients who start the drugs and develop symptomatic brain metastases later on. Most of our patients still received upfront WBRT alone, in contrast to a trend towards primary RS or surgery + RS in the Sperduto et al series. As shown in Table 3, Sperduto et al reported better survival outcomes. Further studies are necessary to evaluate which survival rates are more representative of unselected patient groups in different parts of the world and resource settings.

As shown in Figure 1, the Melanoma-molGPA predicts survival in a relatively reproducible manner. Most survival curves separated excellently. The fact that true long-term survivors were lacking in the most favorable group is probably a 
Table 3. Comparison of the Median Survival Results in Months With Those Reported by Sperduto et al [11, 12]

\begin{tabular}{lllllll}
\hline Study & n & All patients & DS-GPA unfavorable & DS-GPA intermediate & DS-GPA second best & DS-GPA best \\
\hline Sperduto et al [11] & 483 & 6.7 & 3.4 & 4.7 & 8.8 & 13.2 \\
Nieder et al [3] & 51 & 3.7 & 3.1 & 3.7 & 7.5 & 12.7 \\
Sperduto et al [12] & 823 & 9.8 & 4.9 & 8.3 & 15.8 & 34.1 \\
Present study & 69 & 5.4 & 2.1 & 7.8 & 11.8 & 18.0 \\
\hline
\end{tabular}

DS-GPA: diagnosis-specific graded prognostic assessment score (in this case limited to patients with malignant melanoma).

result of the small sample size. Overall, the present data support the use of the four-tiered Melanoma-molGPA.

\section{Conflict of Interest}

The authors declare no conflict of interest.

\section{References}

1. Fife KM, Colman MH, Stevens GN, Firth IC, Moon D, Shannon KF, Harman R, et al. Determinants of outcome in melanoma patients with cerebral metastases. J Clin Oncol. 2004;22(7):1293-1300.

2. Goyal S, Silk AW, Tian S, Mehnert J, Danish S, Ranjan S, Kaufman HL. Clinical management of multiple melanoma brain metastases: a systematic review. JAMA Oncol. 2015;1(5):668-676.

3. Nieder C, Marienhagen K, Geinitz H, Grosu AL. Can current prognostic scores reliably guide treatment decisions in patients with brain metastases from malignant melanoma? J Cancer Res Ther. 2011;7(1):47-51.

4. Gaudy-Marqueste C, Dussouil AS, Carron R, Troin L, Malissen N, Loundou A, Monestier S, et al. Survival of melanoma patients treated with targeted therapy and immunotherapy after systematic upfront control of brain metastases by radiosurgery. Eur J Cancer. 2017;84:44-54.

5. Samlowski WE, Watson GA, Wang M, Rao G, Klimo P, Jr., Boucher K, Shrieve DC, et al. Multimodality treatment of melanoma brain metastases incorporating stereotactic radiosurgery (SRS). Cancer. 2007;109(9):18551862.

6. Radbill AE, Fiveash JF, Falkenberg ET, Guthrie BL, Young PE, Meleth S, Markert JM. Initial treatment of melanoma brain metastases using gamma knife radiosurgery: an evaluation of efficacy and toxicity. Cancer. 2004;101(4):825-833.

7. Raizer JJ, Hwu WJ, Panageas KS, Wilton A, Baldwin DE, Bailey E, von Althann C, et al. Brain and leptomeningeal metastases from cutaneous melanoma: survival outcomes based on clinical features. Neuro Oncol. 2008;10(2):199207.

8. Vennepureddy A, Thumallapally N, Motilal Nehru V, Atallah JP, Terjanian T. Novel drugs and combination therapies for the treatment of metastatic melanoma. J Clin Med Res. 2016;8(2):63-75.

9. Nieder C, Mehta MP. Prognostic indices for brain metastases--usefulness and challenges. Radiat Oncol. 2009;4:10.

10. Sperduto PW, Berkey B, Gaspar LE, Mehta M, Curran W. A new prognostic index and comparison to three other indices for patients with brain metastases: an analysis of 1,960 patients in the RTOG database. Int J Radiat Oncol Biol Phys. 2008;70(2):510-514.

11. Sperduto PW, Chao ST, Sneed PK, Luo X, Suh J, Roberge D, Bhatt A, et al. Diagnosis-specific prognostic factors, indexes, and treatment outcomes for patients with newly diagnosed brain metastases: a multi-institutional analysis of 4,259 patients. Int J Radiat Oncol Biol Phys. 2010;77(3):655-661.

12. Sperduto PW, Jiang W, Brown PD, Braunstein S, Sneed P, Wattson DA, Shih HA, et al. Estimating survival in melanoma patients with brain metastases: an update of the graded prognostic assessment for melanoma using molecular markers (Melanoma-molGPA). Int J Radiat Oncol Biol Phys. 2017;99(4):812-816.

13. Nieder C, Hintz M, Oehlke O, Bilger A, Grosu AL. Validation of the graded prognostic assessment for lung cancer with brain metastases using molecular markers (lungmolGPA). Radiat Oncol. 2017;12(1):107.

14. Nieder C, Hintz M, Grosu AL. Colorectal cancer metastatic to the brain: analysis of prognostic factors and impact of KRAS mutations on presentation and outcome. Clin Transl Oncol. 2016;18(1):88-92.

15. Feng R, Oermann EK, Shrivastava R, Gold A, Collins BT, Kondziolka D, Collins SP. Stereotactic radiosurgery for melanoma brain metastases: a comprehensive clinical case series. World Neurosurg. 2017;100:297-304.

16. Frinton E, Tong D, Tan J, Read G, Kumar V, Kennedy $\mathrm{S}$, Lim C, et al. Metastatic melanoma: prognostic factors and survival in patients with brain metastases. J Neurooncol. 2017;135(3):507-512. 